\title{
Harrison transformation of hyperelliptic solutions and charged dust disks
}

\author{
C. Klein, Max-Planck-Institut für Physik, Föhringer Ring 6, 80805 München, Germany
}

November 18, 2018

\begin{abstract}
We use a Harrison transformation on solutions to the stationary axisymmetric Einstein equations to generate solutions of the Einstein-Maxwell equations. The case of hyperelliptic solutions to the Ernst equation is studied in detail. Analytic expressions for the metric and the multipole moments are obtained. As an example we consider the transformation of a family of counter-rotating dust disks. The resulting solutions can be interpreted as disks with currents and matter with a purely azimuthal pressure or as two streams of freely moving charged particles. We discuss interesting limiting cases as the extreme limit where the charge becomes identical to the mass, and the ultrarelativistic limit where the central redshift diverges.
\end{abstract}

\section{Introduction}

Electro-magnetic fields, especially magnetic fields are of astrophysical importance in the context of neutron stars, white dwarfs and galaxy formation. To get a complete relativistic understanding of such situations, one has to study the coupled Einstein-Maxwell equations. Since the stationary axisymmetric Einstein-Maxwell equations in vacuum in the form of Ernst [1] are completely integrable (see [2]), powerful solution generating techniques are at hand to obtain physically interesting solutions. But the equations in the matter region do not seem to be integrable, which makes it difficult to find global solutions which hold both in a three-dimensionally extended matter region and in vacuum.

In the case of infinitesimally thin disks, the equations in the matter region reduce to ordinary differential equations the solutions of which determine boundary data for the vacuum equations. Such disks are discussed in astrophysics as models for Newtonian galaxies and the matter in accretion disks around black holes. Bičák, Lynden-Bell and Katz [3] studied static spacetimes, from which they removed a strip and glued the remainder together at the equatorial plane. The non-continuous normal derivatives at the equatorial plane lead to a $\delta$-type energy-momentum tensor which can be interpreted as an infinitely extended disk made up of counter-rotating dust. This method was extended in 4] to generate disk sources of the Kerr-metric. With the same techniques, disk sources for Kerr-Newman metrics [5], static axisymmetric spacetimes with magnetic fields [6] and conformastationary metrics [7] were given. By construction all these disks have an infinite extension but finite mass.

In the present paper we give solutions to the Einstein-Maxwell equations with disk sources of finite extension. The solutions are obtained by exploiting the $S U(2,1)$-invariance of the stationary vacuum Einstein-Maxwell equations (see [8] to [12]). We use a so-called Harrison transformation [13] on disk solutions on hyperelliptic Riemann surfaces for the pure Einstein case, which are given in terms of Korotkin's 14 theta functional solutions, to obtain charged disks with currents. In this case analytic formulas for the complete metric can be obtained. As an example we discuss the transformation of a family of counter-rotating disks [15] which contains the Bardeen-Wagoner disk [16] (see [17] for the exact solution) as a limiting case. Following [5] the resulting disk can be interpreted either as a disk with a purely azimuthal pressure and currents or in a certain range of the physical parameters as two streams of freely moving charged particles, i.e. which move on electro-geodesics (solution to the geodesic equation 
in the presence of a Lorentz force). We discuss the energy-momentum tensor at the disk, the multipole moments and interesting limiting cases.

The paper is organized as follows. In section 2 we collect results on the stationary axisymmetric case in the absence of electromagnetic fields, solutions on hyperelliptic Riemann surfaces, and the family of disks discussed in [15, 18. The Einstein-Maxwell equations, their group structure and the action of Harrison transformations on the multipoles are discussed in section 3. In section 4 we consider a Harrison transformation of the counter-rotating disks [15] and discuss the metric and the energy-momentum tensor at the disk. The results are summarized in section 5 together with some concluding remarks.

\section{Stationary axisymmetric Einstein equations, theta functional solutions and counter-rotating dust disks}

In this section we will summarize results on the integrability of the Ernst equation, a class of solutions on hyperelliptic Riemann surfaces [14] and a member of this class describing counter-rotating dust disks which were discussed in 19] and 18.

\subsection{Ernst potential and metric}

We use the Weyl-Lewis-Papapetrou metric (see e.g. 20]

$$
\mathrm{d} s^{2}=-e^{2 U}(\mathrm{~d} t+a \mathrm{~d} \phi)^{2}+e^{-2 U}\left(e^{2 k}\left(\mathrm{~d} \rho^{2}+\mathrm{d} \zeta^{2}\right)+\rho^{2} \mathrm{~d} \phi^{2}\right),
$$

where $\rho$ and $\zeta$ are Weyl's canonical coordinates and $\partial_{t}$ and $\partial_{\phi}$ are the two commuting asymptotically timelike respectively spacelike Killing vectors. With $\xi=\zeta-i \rho$, we define the complex Ernst potential $\mathcal{E}=f+i b$ which is subject to the Ernst equation [21]

$$
\mathcal{E}_{\xi \bar{\xi}}-\frac{1}{2(\bar{\xi}-\xi)}\left(\mathcal{E}_{\bar{\xi}}-\mathcal{E}_{\xi}\right)=\frac{2}{\mathcal{E}+\overline{\mathcal{E}}} \mathcal{E}_{\xi} \mathcal{E}_{\bar{\xi}}
$$

where a bar denotes complex conjugation in $\mathbb{C}$. If the Ernst potential is real, the corresponding spacetime is static and belongs to the so-called Weyl class. For a given Ernst potential the metric (11) follows from $e^{2 U}=f$ and

$$
\begin{aligned}
& a_{\xi}=2 \rho \frac{(\mathcal{E}-\overline{\mathcal{E}})_{\xi}}{(\mathcal{E}+\overline{\mathcal{E}})^{2}} \\
& k_{\xi}=(\xi-\bar{\xi}) \frac{\mathcal{E}_{\xi} \overline{\mathcal{E}}_{\xi}}{(\mathcal{E}+\overline{\mathcal{E}})^{2}} .
\end{aligned}
$$

Thus the complete metric can be obtained from a given Ernst potential via quadratures.

Solutions with equatorial symmetry, i.e. a class of solutions where the metric functions have a reflection symmetry at the equatorial plane $\zeta=0$ (this implies $\mathcal{E}(-\zeta)=\overline{\mathcal{E}}(\zeta)$ ), are of special physical interest. In a Newtonian setting it can be proven that perfect fluids in thermodynamical equilibrium lead to equatorially symmetric situations, and the same is assumed to hold in a general relativistic context. A consequence of this condition is that Newman-Unti-Tambourini-parameters are ruled out.

The stationary axisymmetric Einstein equations in vacuum were shown to be completely integrable in [22] and [23. This implies that the Ernst equation has an infinite dimensional symmetry group, the Geroch group (see [24]). The equation can be treated as the integrability condition for an overdetermined linear differential system for a $2 \times 2$-matrix $\Psi$ (see [25])

$$
\begin{aligned}
& \Psi_{\xi} \Psi^{-1}=\left(\begin{array}{cc}
\mathcal{M} & 0 \\
0 & \mathcal{N}
\end{array}\right)+\sqrt{\frac{K-\xi}{K-\bar{\xi}}}\left(\begin{array}{cc}
0 & \mathcal{M} \\
\mathcal{N} & 0
\end{array}\right) \\
& \Psi_{\bar{\xi}} \Psi^{-1}=\left(\begin{array}{cc}
\overline{\mathcal{N}} & 0 \\
0 & \overline{\mathcal{M}}
\end{array}\right)+\sqrt{\frac{K-\xi}{K-\bar{\xi}}}\left(\begin{array}{cc}
0 & \overline{\mathcal{N}} \\
\overline{\mathcal{M}} & 0
\end{array}\right) ;
\end{aligned}
$$


here $K$ is the so-called spectral parameter, and

$$
\mathcal{M}=\frac{\overline{\mathcal{E}}_{\xi}}{\mathcal{E}+\overline{\mathcal{E}}}, \quad \mathcal{N}=\frac{\mathcal{E}_{\xi}}{\mathcal{E}+\overline{\mathcal{E}}}
$$

The spectral parameter is defined on the family of Riemann surfaces $\Sigma_{0}$ given by the algebraic relation between $\mu_{0}$ and $K$,

$$
\mu_{0}^{2}=(K-\xi)(K-\bar{\xi})
$$

Notice the special feature of the Ernst equation that the branch points $\xi, \bar{\xi}$ depend on the spacetime coordinates.

It is a consequence of the linear system (5) that a matrix $\Psi$ considered as a function on $\Sigma_{0}$ and subject to the following conditions leads to a solution to the Ernst equation:

I. The matrix $\Psi$ is at least twice differentiable with respect to $\xi$ and $\bar{\xi}$. $\Psi(P)$ is holomorphic and invertible at the branch points $\xi$ and $\bar{\xi}$ such that the logarithmic derivative $\Psi_{\xi} \Psi^{-1}$ has a pole at $\xi$ and $\Psi_{\bar{\xi}} \Psi^{-1}$ has a pole at $\bar{\xi}$.

II. All singularities of $\Psi$ on $\Sigma_{0}$ are such that the logarithmic derivatives $\Psi_{\xi} \Psi^{-1}$ and $\Psi_{\bar{\xi}} \Psi^{-1}$ are holomorphic there.

III. $\Psi$ is subject to the reduction condition

$$
\Psi\left(P^{\sigma_{0}}\right)=\sigma_{3} \Psi(P) \sigma_{2}
$$

where $\sigma_{2}, \sigma_{3}$ are Pauli matrices, and where $\sigma_{0}$ denotes the involution on $\Sigma_{0}$ which interchanges the sheets. IV. The normalization and reality condition

$$
\Psi\left(P=\infty^{+}\right)=\left(\begin{array}{cc}
\overline{\mathcal{E}} & -i \\
\mathcal{E} & i
\end{array}\right)
$$

The function $\mathcal{E}$ in (9) is a solution to the Ernst equation.

We note that the choice of $\sigma_{2}=\left(\begin{array}{cc}0 & -i \\ i & 0\end{array}\right)$ in condition III is a gauge condition, which does not fix the gauge completely, however. The remaining gauge freedom is due to matrices $C(K)=\kappa_{1}(K) \hat{1}+\kappa_{2}(K) \sigma_{2}$, where the $\kappa_{i}$ do not depend on $\xi, \bar{\xi}$ and obey the asymptotic conditions $\kappa_{1}(\infty)=1$ and $\kappa_{2}(\infty)=0$. The matrices $C$ act on $\Psi$ in the form $\Psi \rightarrow \Psi C(K)$. Since it is a consequence of (15) that $\operatorname{det} \Psi=F(K) e^{2 U}$ where $F(K)$ is independent of $\xi, \bar{\xi}$ we can use this gauge freedom to choose $F(K)=1$. The linear system (15) leads for the matrix $\chi(P)=\Psi^{-1}\left(\infty^{-}\right) \Psi(P)$ to one of the linear systems used in [24]. This parametrization, especially

$$
\Psi^{-1}\left(\infty^{-}\right) \Psi\left(\infty^{+}\right)=\frac{1}{\mathcal{E}+\overline{\mathcal{E}}}\left(\begin{array}{cc}
2 \mathcal{E} \overline{\mathcal{E}} & i(\overline{\mathcal{E}}-\mathcal{E}) \\
i(\overline{\mathcal{E}}-\mathcal{E}) & 2
\end{array}\right)
$$

reveals that the Ernst equation is invariant under $S U(1,1) / S O(1,1)$ transformations. For more details on the group aspect see [24] and the discussion in the next section.

For given $\Psi(K)$, one can directly determine the metric function $a$ without having to integrate relation (3). We denote by $D_{P} F(P)$ the coefficient of the linear term in the expansion of the function $F$ in the local parameter near $P$. With (5) one finds for the matrix $\mathcal{S}=\Psi^{-1}\left(\infty^{+}\right) D_{\infty^{+}} \Psi\left(\infty^{+}\right)$

$$
\mathcal{S}_{\xi}:=\left(\Psi^{-1}\left(\infty^{+}\right) D_{\infty}+\Psi\right)_{\xi}=\frac{\xi-\bar{\xi}}{2(\mathcal{E}+\overline{\mathcal{E}})^{2}}\left(\begin{array}{cc}
(\mathcal{E} \overline{\mathcal{E}})_{\xi} & i(\overline{\mathcal{E}}-\mathcal{E})_{\xi} \\
i\left(\mathcal{E}^{2} \overline{\mathcal{E}}_{\xi}-\overline{\mathcal{E}}^{2} \mathcal{E}_{\xi}\right) & -(\mathcal{E} \overline{\mathcal{E}})_{\xi}
\end{array}\right)
$$

This implies with (3)

$$
a-a_{0}=-2 \mathcal{S}_{12}=-2\left(\Psi^{-1}\left(\infty^{+}\right) D_{\infty}+\Psi\right)_{12},
$$

where $a_{0}$ is a constant which is fixed by the condition that $a$ vanishes on the regular part of the axis. 


\subsection{Solutions on hyperelliptic surfaces}

Using the integrability of the Ernst equation, Korotkin [25] gave solutions on hyperelliptic Riemann surfaces $\Sigma_{g}$ of genus $g$ which are defined by the algebraic relation

$$
\mu^{2}=(K-\xi)(K-\bar{\xi}) \prod_{i=1}^{g}\left(K-E_{i}\right)\left(K-\bar{E}_{i}\right)
$$

The points $E_{i}=\alpha_{i}-i \beta_{i}$ have to be constant with respect to the physical coordinates. We order the branch points with $\operatorname{Im} E_{i}<0$ in a way that $\Re E_{1}<\Re E_{2}<\ldots<\Re E_{g}$ and assume for simplicity that the real parts of the $E_{i}$ are all different. On this surface we introduce a canonical basis of cycles $\left(a_{i}, b_{i}\right), i=1, \ldots, g$, the $g$ differentials of the first kind $\mathrm{d} \omega_{i}$ normalized by the condition $\oint_{a_{i}} \mathrm{~d} \omega_{j}=2 \pi \mathrm{i} \delta_{i j}$, and the Abel map $\omega_{i}(P)=\int_{P_{0}}^{P} \mathrm{~d} \omega_{i}$. Furthermore, we define the Riemann matrix $\Pi$ with the elements $\pi_{i j}=\oint_{b_{i}} \mathrm{~d} \omega_{j}$, and the theta function

$$
\Theta[m](z)=\sum_{N \in Z^{g}} \exp \left\{\frac{1}{2}\left\langle\Pi\left(N+\frac{m^{1}}{2}\right),\left(N+\frac{m^{1}}{2}\right)\right\rangle+\left\langle\left(z+\pi i m^{2}\right),\left(N+\frac{\alpha}{2}\right)\right\rangle\right\}
$$

with half integer characteristic $[m]=\left[\begin{array}{c}m^{1} \\ m^{2}\end{array}\right]$ and $m_{i}^{1}, m_{i}^{2}=0,1\left(\langle N, z\rangle=\sum_{i=1}^{g} N_{i} z_{i}\right)$. We write $\Theta$ for the theta function with characteristic [0], and $P=(K, \pm \mu(K))$ or $K^{ \pm}$for a point $P \in \Sigma_{g}$ (the + sheet will be defined by the condition that $\lim _{K \rightarrow \infty} \mu(K) / K^{g}=+1$ and similarly for the --sheet). The normalized (all $a$-periods zero) differential of the third kind with poles at $P_{1}$ and $P_{2}$ and residue +1 and -1 respectively will be denoted by $\mathrm{d} \omega_{P_{1} P_{2}}$.

Let $\ln G(K)$ be a Hölder-continuous function subject to the condition $G(\bar{K})=\bar{G}(K)$ and

$$
I=\frac{1}{2 \pi i} \int_{\Gamma} \ln G(\tau) d \omega_{\infty^{+} \infty^{-}}(\tau), \quad u_{i}=\frac{1}{2 \pi i} \int_{\Gamma} \ln G d \omega_{i}
$$

where $\Gamma$ is the covering of the imaginary axis in the + -sheet of $\Sigma_{g}$ between $-i$ and $i$. Then one obtains solutions to the Ernst equation in the form

$$
\mathcal{E}(\rho, \zeta)=\frac{\Theta[m]\left(\omega\left(\infty^{+}\right)+u+\omega(\bar{\xi})\right)}{\Theta[m]\left(\omega\left(\infty^{+}\right)-u+\omega(\bar{\xi})\right)} e^{I} .
$$

Notice that these solutions depend only via the branch points of $\Sigma_{g}$ on the physical coordinates. In [27] it was shown that solutions of the above form on a Riemann surface of even genus $g=2 s$ given by $\mu^{2}=(K-\xi)(K-\bar{\xi}) \prod_{i=1}^{s}\left(K^{2}-E_{i}^{2}\right)\left(K^{2}-\bar{E}_{i}^{2}\right)$ with a function $G$ subject to $G(-K)=\bar{G}(K)$ lead to an equatorially symmetric Ernst potential.

The Ernst potential (16) follows with (9) and $J=\frac{1}{2 \pi i} \int_{\Gamma} \ln G d \omega_{P P^{\sigma}}$ from a matrix $\Psi$ of the form

$$
\Psi=e^{I / 2} \sqrt{\frac{\operatorname{det}(\infty)}{\operatorname{det}(K)}}\left(\begin{array}{cc}
\frac{\Theta[m](u+\omega(P))}{\Theta[m](u+\omega(\infty)-))} e^{J / 2} & -i \frac{\Theta[m]\left(u+\omega\left(P^{\sigma}\right)\right)}{\Theta[m]\left(u+\omega\left(\infty^{-}\right)\right)} e^{-J / 2} \\
\frac{\Theta[m](u+\omega(P)+\omega(\bar{\xi}))}{\Theta[m]\left(u+\omega\left(\infty^{-}\right)+\omega(\xi)\right)} e^{J / 2} & i \frac{\Theta[m]\left(u+\omega\left(P^{\sigma}\right)+\omega(\xi)\right)}{\Theta[m]\left(u+\omega\left(\infty^{-}\right)+\omega(\xi)\right)} e^{-J / 2}
\end{array}\right),
$$

with $\operatorname{det}(K)=\Theta[m](u+\omega(P)) \Theta[m](u-\omega(P)+\omega(\bar{\xi}))+\Theta[m](u-\omega(P)) \Theta[m](u+\omega(P)+\omega(\bar{\xi}))$.

To determine the metric function $a$ via (12), we have to calculate the matrix $\mathcal{S}$ which leads with (17) to

$$
\mathcal{S}_{12}=\frac{1}{2 i f} D_{\infty^{+}} \ln \frac{\Theta\left(u+\omega\left(\infty^{-}\right)\right)}{\Theta\left(u+\omega\left(\infty^{-}\right)+\omega(\bar{\xi})\right)},
$$

and to determine the Harrison transformed function $a$ in the next section,

$$
\mathcal{S}_{21}=-\frac{\mathcal{E} \overline{\mathcal{E}}}{2 i f} D_{\infty^{+}} \ln \frac{\Theta\left(u+\omega\left(\infty^{+}\right)\right)}{\Theta\left(u+\omega\left(\infty^{+}\right)+\omega(\bar{\xi})\right)} .
$$


Using a degenerated version of Fay's trisecant identity [26] (see 27] for the present case), we can write the above relations free of derivatives,

$$
\begin{aligned}
S_{12} & =\frac{\rho}{2 f}\left(\frac{\Theta[m](u) \Theta[m]\left(u+2 \omega\left(\infty^{-}\right)+\omega(\bar{\xi})\right)}{L \Theta[m]\left(u+\omega\left(\infty^{-}\right)+\omega(\bar{\xi})\right) \Theta[m]\left(u+\omega\left(\infty^{-}\right)\right)}-1\right) \\
S_{21} & =-\frac{\rho \mathcal{E} \overline{\mathcal{E}}}{2 f}\left(\frac{\Theta[m](u) \Theta[m]\left(u+2 \omega\left(\infty^{+}\right)+\omega(\bar{\xi})\right)}{L \Theta[m]\left(u+\omega\left(\infty^{+}\right)+\omega(\bar{\xi})\right) \Theta[m]\left(u+\omega\left(\infty^{+}\right)\right)}-1\right),
\end{aligned}
$$

where

$$
L=\frac{\Theta\left(\omega\left(\infty^{-}\right)\right) \Theta\left(\omega\left(\infty^{-}\right)+\omega(\bar{\xi})\right)}{\Theta(0) \Theta(\omega(\bar{\xi}))} .
$$

To construct the solution for the counter-rotating disks in 19, we used an algebraic approach which made it possible to establish algebraic relations between the metric functions at the disk. Let us recall that a divisor $X$ on $\Sigma_{g}$ is a formal symbol $X=n_{1} P_{1}+\ldots+n_{k} P_{k}$ with $P_{i} \in \Sigma$ and $n_{i} \in \mathrm{Z}$. The degree of a divisor is $\sum_{i=1}^{k} n_{i}$. The Riemann vector $K_{R}$ is defined by the condition that $\Theta\left(\omega(W)+K_{R}\right)=0$ if $W$ is a divisor of degree $g-1$ or less. We use here and in the following the notation $\omega(W)=\int_{P_{0}}^{W} \mathrm{~d} \omega=\sum_{i=1}^{g-1} \omega\left(W_{i}\right)$. Note that the Riemann vector can be expressed through half-periods in the case of a hyperelliptic surface. We define the divisor $X=\sum_{i=1}^{g} X_{i}$ as the solution of the Jacobi inversion problem $(i=1, \ldots, g)$

$$
\omega(X)-\omega(D)=u,
$$

where the divisor $D=\sum_{i=1}^{g} E_{i}$ (this corresponds to a choice of the characteristic $[m]$ in (16]). With the help of these divisors, we can write (16) in the form

$$
\ln \mathcal{E}=\int_{D}^{X} \frac{\tau^{g} d \tau}{\mu(\tau)}-\frac{1}{2 \pi \mathrm{i}} \int_{\Gamma} \ln G \frac{\tau^{g} d \tau}{\mu(\tau)}
$$

Additional information follows from the reality of $u$ which leads to $\omega(X)-\omega(D)=\omega(\bar{X})-\omega(\bar{D})$. The reality condition for $X$ implies via Abel's theorem the existence of a meromorphic function $R$ with poles in $\bar{X}+D$ and zeros in $X+\bar{D}$ (which is a rational function in the fundamental polygon),

$$
R(K)=\mathrm{const} \frac{\prod_{i=1}^{g}\left(K-E_{i}\right)\left(K-\overline{\mathcal{E}}_{i}\right)-Q_{0}(K) \mu(K)}{\prod_{i=1}^{g}\left(K-\bar{X}_{i}\right)\left(K-E_{i}\right)},
$$

where $Q_{0}(K)=x_{0}+x_{1} K+\ldots+x K^{g-1}$ is a polynomial in $K$ with purely imaginary coefficients and $x=i b e^{-2 U}$. The coefficients $x_{i}$ are related to $X$ via the relation

$$
\left(1-x^{2}\right) \prod_{i=1}^{g}\left(K-X_{i}\right)\left(K-\bar{X}_{i}\right)=\prod_{i=1}^{g}\left(K-E_{i}\right)\left(K-\overline{\mathcal{E}}_{i}\right)-Q_{0}^{2}(K)(K-\xi)(K-\bar{\xi}) .
$$

We can use the existence of the rational function $R$ to calculate certain integrals of the third kind as

$$
\frac{\Theta(u+\omega(P)) \Theta\left(u+\omega\left(P^{\sigma}\right)+\omega(\bar{\xi})\right)}{\Theta\left(u+\omega\left(P^{\sigma}\right)\right) \Theta(u+\omega(P)+\omega(\bar{\xi}))}=\exp \left(\int_{X+\bar{D}}^{\bar{X}+D} d \omega_{P^{\sigma} P}\right)=\frac{R(P)}{R\left(P^{\sigma}\right)} .
$$

This makes it possible to give an algebraic expression for $S_{12}$ and $S_{21}$. We can write $S_{12}$ in the form

$$
S_{12}=\frac{1}{i(\mathcal{E}+\overline{\mathcal{E}})} D_{\infty^{+}}\left(\int_{X}^{\bar{X}} d \omega_{P Q}\right)=\frac{1}{i(\mathcal{E}+\overline{\mathcal{E}})} D_{\infty^{+}}\left(\int_{X+\bar{D}}^{\bar{X}+D} d \omega_{P Q}+\int_{D}^{\bar{D}} d \omega_{P Q}\right)
$$

with $Q$ independent of $P$. The second integral can be reexpressed in terms of theta functions and be calculated with the help of so-called root functions: The quotient of two theta functions with the same argument but different characteristic is a root function which means that its square is a function on $\Sigma_{g}$. 
Let $P_{i}, i=1, \ldots, 2 g+2$, be the branch points of a hyperelliptic Riemann surface $\Sigma_{g}$ of genus $g$ and $A_{j}=\omega\left(P_{j}\right)$ with $\omega\left(P_{1}\right)=0$. Furthermore let $\left\{i_{1}, \ldots, i_{g}\right\}$ and $\left\{j_{1}, \ldots, j_{g}\right\}$ be two sets of numbers in $\{1,2, \ldots, 2 g+2\}$. Then the following equality holds for an arbitrary point $P \in \Sigma_{g}$,

$$
\frac{\Theta\left[K_{R}+\sum_{k=1}^{g} A_{i_{k}}\right](\omega(P))}{\Theta\left[K_{R}+\sum_{k=1}^{g} A_{j_{k}}\right](\omega(P))}=c_{1} \sqrt{\frac{\left(K-E_{i_{1}}\right) \ldots\left(K-E_{i_{g}}\right)}{\left(K-E_{j_{1}}\right) \ldots\left(K-E_{j_{g}}\right)}},
$$

where $c_{1}$ is a constant independent of $K$.

This implies

$$
\exp \left(\int_{D}^{\bar{D}} d \omega_{P^{\sigma} \infty^{+}}\right)= \pm \prod_{i=1}^{g} \sqrt{\frac{K-F_{i}}{K-E_{i}}}
$$

Thus we get with (26)

$$
\mathcal{S}_{12}=\frac{1}{i(\mathcal{E}+\overline{\mathcal{E}})}\left(\frac{1}{2} \sum_{i=1}^{g}\left(X_{i}-\bar{X}_{i}\right)+\frac{1}{1-x^{2}}\left(\frac{x}{2}\left(\sum_{i=1}^{g}\left(E_{i}+\bar{E}_{i}\right)-(\xi+\bar{\xi})\right)+x_{g-2}\right)\right),
$$

and similarly

$$
\mathcal{S}_{21}=-\frac{\mathcal{E} \overline{\mathcal{E}}}{i(\mathcal{E}+\overline{\mathcal{E}})}\left(\frac{1}{2} \sum_{i=1}^{g}\left(X_{i}-\bar{X}_{i}\right)-\frac{1}{1-x^{2}}\left(\frac{x}{2}\left(\sum_{i=1}^{g}\left(E_{i}+\bar{E}_{i}\right)-(\xi+\bar{\xi})\right)+x_{g-2}\right)\right) .
$$

\subsection{Counter-rotating dust disk}

We summarize results of [19] and [18] on disks which can be interpreted as two counter-rotating components of pressureless matter, so-called dust. The surface energy-momentum tensor $S^{\alpha \beta}$ of these models, where $\alpha$ and $\beta$ stand for the $t, \rho$ and $\phi$ components, is defined on the hypersurface $\zeta=0$. The tensor $S^{\alpha \beta}$ is related to the energy-momentum tensor $T^{\alpha \beta}$ which appears in the Einstein equations $G^{\mu \nu}=8 \pi T^{\mu \nu}$ (we use units in which the Newtonian gravitational constant, the dielectric constant and the velocity of light are equal to 1) via $T^{\alpha \beta}=S^{\alpha \beta} e^{k-U} \delta(\zeta)$. The tensor $S^{\alpha \beta}$ can be written in the form

$$
S^{\alpha \beta}=\sigma_{+} u_{+}^{\alpha} u_{+}^{\beta}+\sigma_{-} u_{-}^{\alpha} u_{-}^{\beta},
$$

where $u_{ \pm}=(1,0, \pm \Omega)$. We gave an explicit solution for disks with constant angular velocity $\Omega$ and constant relative density $\gamma=\left(\sigma_{+}-\sigma_{-}\right) /\left(\sigma_{+}+\sigma_{-}\right)$. This class of solutions is characterized by two real parameters $\lambda$ and $\delta$ which are related to $\Omega$ and $\gamma$ and the metric potential $U_{0}=U(0,0)$ at the center of the disk via

$$
\lambda=2 \Omega^{2} e^{-2 U_{0}}
$$

and

$$
\delta=\frac{1-\gamma^{2}}{\Omega^{2}}
$$

We put the radius $\rho_{0}$ of the disk equal to 1 unless otherwise noted. Since the radius appears only in the combinations $\rho / \rho_{0}, \zeta / \rho_{0}$ and $\Omega \rho_{0}$ in the physical quantities, it does not have an independent role. It is always possible to use it as a natural length scale unless it tends to 0 as in the case of the ultrarelativistic limit of the one component disk. The Ernst potential will be discussed in dependence of the parameters $\epsilon=z_{R} /\left(1+z_{R}\right)=1-e^{U_{0}}$ and $\gamma$, where $z_{R}$ is the redshift of photons emitted at the center of the disk and detected at infinity.

The solution is given on a surface of genus 2 where the branch points of the Riemann surface are given by the relation $E_{1}=-\bar{E}_{2}$ and $E:=E_{1}^{2}=\alpha+\mathrm{i} \beta$ with $\alpha, \beta$ given by

$$
\alpha=-1+\frac{\delta}{2}, \quad \beta=\sqrt{\frac{1}{\lambda^{2}}+\delta-\frac{\delta^{2}}{4}} .
$$


The function $G$ in (16) reads

$$
G(\tau)=\frac{\sqrt{\left(\tau^{2}-\alpha\right)^{2}+\beta^{2}}+\tau^{2}+1}{\sqrt{\left(\tau^{2}-\alpha\right)^{2}+\beta^{2}}-\left(\tau^{2}+1\right)} .
$$

We note that with $\alpha$ and $\beta$ given, the Riemann surface is completely determined at a given point in the spacetime, i.e. for a given value of $\xi$.

Regularity of the solutions in the exterior of the disk restricts the physical parameters to $0 \leq \delta \leq \delta_{s}(\lambda):=$ $2\left(1+\sqrt{1+1 / \lambda^{2}}\right)$ and $0<\lambda \leq \lambda_{c}$ where $\lambda_{c}(\gamma)$ is the smallest value of $\lambda$ for which $\epsilon=1$. The range of the physical parameters is restricted by the following limiting cases:

Newtonian limit: $\epsilon=0(\lambda=0)$, i.e. small velocities $\Omega \rho_{0}$ and small redshifts in the disk. The function $e^{2 U}$ tends independently of $\gamma$ to $1+\lambda U_{N}$, where $U_{N}$ is the Maclaurin disk solution, and $b$ is of order $\Omega^{3}$. Ultrarelativistic limit: $\epsilon=1$, i.e. diverging central redshift. For $\gamma \neq 1$ it is reached for $\lambda_{c}=\infty$. The solution describes a disk of finite extension with diverging central redshift. For $\gamma=1$, the limit is reached for $\lambda_{c}=4.629 \ldots$. In this case the solution has a singular axis and is not asymptotically flat. This behavior can be interpreted as the limit of a vanishing disk radius. With this rescaling the solution in the exterior of the disk can be interpreted as the extreme Kerr solution (see [18] and references given therein).

Static limit: $\gamma=0\left(\delta=\delta_{s}(\lambda)\right)$. In this limit, the solution belongs to the Morgan and Morgan class [29]. One component: $\gamma=1(\delta=0)$, i.e. no counter-rotating matter in the disk. This is the disk of [16], [17]. Analytic formulas for the complete metric in terms of theta functions are given in [18]. To evaluate the hyperelliptic integrals in the expressions for the metric we use the numerical methods and the cut-system of 18 .

At the disk the branch points $\xi, \bar{\xi}$ lie on the contour $\Gamma$ which implies that care has to be taken in the evaluation of the line integrals. The situation is however simplified by the equatorial symmetry of the solution which is reflected by the additional involution $K \rightarrow-K$ of the Riemann surface $\Sigma_{2}$ for $\zeta=0$. This makes it possible to perform the reduction $K^{2} \rightarrow \tau$ and to express the metric in terms of elliptic theta functions (see [27]). We denote with $\Sigma_{w}$ the elliptic Riemann surface defined by $\mu_{w}^{2}=\left(\tau+\rho^{2}\right)\left((\tau-\alpha)^{2}+\beta^{2}\right)$, and let $d w$ be the associated differential of the first kind with $u_{w}=$ $\frac{1}{i \pi} \int_{-\rho^{2}}^{-1} \ln G(\sqrt{\tau}) d w(\tau)$. We cut the surface in a way that the $a$-cut is a closed contour in the upper sheet around the cut $\left[-\rho^{2}, \bar{E}\right]$ and that the $b$-cut starts at the cut $[\infty, E]$. The Abel map $w$ is defined for $P \in \Sigma_{w}$ as $w(P)=\int_{\infty}^{P} d w$. Then the real part of the Ernst potential at the disk can be written as

$$
\begin{aligned}
e^{2 U}= & \frac{1}{Y-\delta}\left(-\frac{1}{\lambda}-\frac{Y}{\delta}\left(\frac{\frac{1}{\lambda^{2}}+\delta}{\sqrt{\frac{1}{\lambda^{2}}+\delta \rho^{2}}}-\frac{1}{\lambda}\right)\right. \\
& \left.+\sqrt{\frac{Y^{2}\left(\left(\rho^{2}+\alpha\right)^{2}+\beta^{2}\right)}{\frac{1}{\lambda^{2}}+\delta \rho^{2}}-2 Y\left(\rho^{2}+\alpha\right)+\frac{1}{\lambda^{2}}+\delta \rho^{2}}\right)
\end{aligned}
$$

where

$$
Y=\frac{\frac{1}{\lambda^{2}}+\delta \rho^{2}}{\sqrt{\left(\rho^{2}+\alpha\right)^{2}+\beta^{2}}} \frac{\vartheta_{3}^{2}\left(u_{w}\right)}{\vartheta_{1}^{2}\left(u_{w}\right)} .
$$

It was shown that there exist algebraic relations between the real and imaginary parts of the Ernst potential,

$$
\frac{\delta^{2}}{2}\left(e^{4 U}+b^{2}\right)=\left(\frac{1}{\lambda}-\delta e^{2 U}\right)\left(\frac{\frac{1}{\lambda^{2}}+\delta}{\sqrt{\frac{1}{\lambda^{2}}+\delta \rho^{2}}}-\frac{1}{\lambda}\right)+\delta\left(\frac{\delta+\rho^{2}}{2}-1\right),
$$

and the function $Z:=\left(a-a_{0}\right) e^{2 U}$

$$
Z^{2}-\rho^{2}+\delta e^{4 U}=\frac{2}{\lambda} e^{2 U}
$$


Moreover we have

$$
i x_{0}=-\frac{Z}{\delta f}\left(\frac{1 / \lambda^{2}+\delta}{\sqrt{1 / \lambda^{2}+\delta \rho^{2}}}-\frac{1}{\lambda}\right) .
$$

At the disk, the normal derivatives of the metric functions are discontinuous which leads via the Einstein equations to a $\delta$-type energy-momentum tensor. To determine this tensor, it seems best to use Israel's invariant junction conditions for matching spacetimes across non-null hypersurfaces [30]. As in [19] we get

$$
\begin{aligned}
s_{1}:=-4 \pi s_{00} & =\left(k_{\zeta}-2 U_{\zeta}\right) e^{2 U}, \\
s_{2}:=-4 \pi\left(s_{03}-a s_{00}\right) & =-\frac{1}{2} a_{\zeta} e^{2 U}, \\
s_{3}:=-4 \pi\left(s_{33}-2 a s_{03}+a^{2} s_{00}\right) & =-k_{\zeta} \rho^{2} e^{-2 U},
\end{aligned}
$$

where we define the tensor $s^{\mu \nu}$ via $T^{\mu \nu}=s^{\mu \nu} e^{2(k-U)} \delta(\zeta)$. The right-hand sides in (42) can be expressed in terms of $\rho$-derivatives in the disk via

$$
\begin{aligned}
\left(e^{2 U}\right)_{\zeta} & =\frac{Z^{2}+\rho^{2}+\delta e^{4 U}}{2 Z \rho} b_{\rho} \\
b_{\zeta} & =-\frac{Z^{2}+\rho^{2}+\delta e^{4 U}}{2 Z \rho}\left(e^{2 U}\right)_{\rho}+\frac{e^{2 U}}{Z} .
\end{aligned}
$$

This makes it directly possible to determine all quantities in the disk in terms of elliptic functions.

\section{Einstein-Maxwell equations and Harrison transformations}

\subsection{Field equations and group structure}

To treat the Einstein-Maxwell equations in vacuum in the stationary axisymmetric case we use again the metric (1). We gauge the electromagnetic 4-potential in a way that it has only non-vanishing $A_{t}$ and $A_{\phi}$ components. It is subject to the Maxwell equations

$$
F_{\mu \nu} ; \nu=0, \quad{ }^{*} F_{\mu \nu} ; \nu=\frac{1}{2} \epsilon_{\mu \nu \alpha \beta} F^{\alpha \beta ; \nu}=0, \quad F_{\mu \nu}=A_{\mu, \nu}-A_{\nu, \mu}
$$

The Einstein equations lead to

$$
R_{\mu \nu}=F_{\mu \lambda} F_{\nu}^{\lambda}-\frac{1}{4} g_{\mu \nu} F_{\kappa \lambda} F^{\kappa \lambda}
$$

The vacuum Einstein-Maxwell equations in the stationary axisymmetric case are equivalent to the complex Ernst equations [1

$$
\begin{aligned}
& f \Delta \mathcal{E}=(\nabla \mathcal{E}+2 \bar{\Phi} \nabla \Phi) \nabla \mathcal{E}, \\
& f \Delta \Phi=(\nabla \mathcal{E}+2 \bar{\Phi} \nabla \Phi) \nabla \Phi,
\end{aligned}
$$

where $\Delta$ and $\nabla$ are the standard differential operators in cylindrical coordinates, where the potentials $\mathcal{E}$ and $\Phi$ are independent of $\phi$, and where $\Re \mathcal{E}=f-\Phi \bar{\Phi}$ and $f=e^{2 U}$. The remaining metric functions follow via

$$
\begin{aligned}
& k_{\xi}=\frac{\xi-\bar{\xi}}{f}\left(\frac{1}{4 f}\left(\mathcal{E}_{\xi}+2 \bar{\Phi} \Phi_{\xi}\right)\left(\overline{\mathcal{E}}_{\xi}+2 \Phi \bar{\Phi}_{\xi}\right)-\Phi_{\xi} \bar{\Phi}_{\xi}\right) \\
& a_{\xi}=\frac{\rho}{f^{2}}\left(\mathrm{i}(\Im \mathcal{E})_{\xi}+\Phi \bar{\Phi}_{\xi}-\bar{\Phi} \Phi_{\xi}\right)
\end{aligned}
$$


from $\mathcal{E}$ and $\Phi$. The electromagnetic potentials are related to $\Phi$ via $A_{t}=\Re \Phi$ and

$$
(\Im \Phi)_{\xi}=\frac{i}{\rho} f\left(A_{\phi, \xi}-a A_{t, \xi}\right) .
$$

For $\Phi=0$ one obtains the pure Einstein case of the previous section.

The system (46) is again integrable and can be treated as the integrability condition of a linear differential system as in the pure Einstein case (see [11). Even without axial symmetry the stationary EinsteinMaxwell equations are equivalent to a $S U(2,1) / S[U(1,1) \times U(1)]$ nonlinear sigma model. This group can be parametrized by trigonal $3 \times 3$ matrices $V$,

$$
V=\left(\begin{array}{ccc}
\sqrt{f} & 0 & 0 \\
i \sqrt{2} \Phi & 1 & 0 \\
\left(b+i|\Phi|^{2}\right) / \sqrt{f} & (\sqrt{2} \bar{\Phi}) / \sqrt{f} & 1 / \sqrt{f}
\end{array}\right)
$$

The matrix $V$ satisfies

$$
V^{\dagger} \eta V=\eta, \quad \eta=\left(\begin{array}{ccc}
0 & 0 & i \\
0 & 1 & 0 \\
-i & 0 & 0
\end{array}\right)
$$

i.e. it is unitary with respect to the metric $\eta$ of $S U(2,1)$. The action of $\mathcal{G} \in S U(2,1)$ on $V$ is $V \rightarrow$ $h(V, \mathcal{G}) V \mathcal{G}^{-1}$ where $h(V, \mathcal{G}) \in S[U(1,1) \times U(1)]$ restores the triangular gauge of $V$. To obtain a gauge invariant parametrization, one introduces $\chi:=\Xi V^{\dagger} \Xi V$ with $\Xi=\operatorname{diag}(1,-1,1)$ on which the action of $\mathcal{G} \in S U(2,1)$ is given by $\chi \rightarrow \Xi\left(\mathcal{G}^{-1}\right)^{\dagger} \Xi \chi \mathcal{G}^{-1}$. We have

$$
\chi=\left(\begin{array}{ccc}
f-2|\Phi|^{2}+\left(b^{2}+|\Phi|^{4}\right) / f & \sqrt{2} \bar{\Phi}\left(b-i|\Phi|^{2}+i f\right) / f & \left(b-i|\Phi|^{2}\right) / f \\
-\sqrt{2} \Phi\left(b+i|\Phi|^{2}-i f\right) / f & 1-2|\Phi|^{2} / f & -\sqrt{2} \Phi / f \\
\left(b+i|\Phi|^{2}\right) / f & \sqrt{2} \bar{\Phi} / f & 1 / f
\end{array}\right) .
$$

The $S U(2,1)$ symmetry can be used to generate solutions by the action of an element $\mathcal{G}$. We list the infinitesimal transformations and their consequences: $\left(\begin{array}{ccc}0 & 0 & 0 \\ \theta_{1} & 0 & 0 \\ \theta_{2} & \theta_{3} & 0\end{array}\right)$ are gauge transformations which add physically irrelevant constants to $\Im \mathcal{E}$ and $\Im \Phi,\left(\begin{array}{lll}0 & 0 & \theta \\ 0 & 0 & 0 \\ 0 & 0 & 0\end{array}\right)$ is an Ehlers transformation which changes $f \rightarrow b,\left(\begin{array}{ccc}i \theta & 0 & 0 \\ 0 & -2 i \theta & 0 \\ 0 & 0 & i \theta\end{array}\right)$ is an electromagnetic duality transformation, $\left(\begin{array}{ccc}\theta & 0 & 0 \\ 0 & 0 & 0 \\ 0 & 0 & -\theta\end{array}\right)$ is a scale transformation, $f, b, \Phi \rightarrow e^{\theta} f, e^{\theta} b, e^{\theta / 2} \Phi$, and $\left(\begin{array}{ccc}0 & -i \theta & 0 \\ 0 & 0 & \bar{\theta} \\ 0 & 0 & 0\end{array}\right)$ is a Harrison transformation [13] which changes $f \rightarrow \Phi$.

\subsection{Harrison transformations}

Here we are interested to generate charged solutions from non-charged ones via a Harrison transformation, i.e. to generate solutions to the Einstein-Maxwell equations containing one additional constant parameter which is related to the charge. We assume that the original solutions are equatorially symmetric and asymptotically flat, i.e. $f \rightarrow 1, \Phi \rightarrow 0$ and $b \rightarrow 0$ for $|z| \rightarrow \infty$. To ensure that the transformed solutions have the same asymptotic behavior, one has to use a scale transformation together with a transformation which changes $\Phi$ and $b$ by some constant. By exponentiating the matrices of the $S U(2,1)$ transformations, we consider a transformation of the form

$$
\mathcal{G}=\left(\begin{array}{ccc}
1 & i \theta_{1} & -i \theta_{1} \overline{\theta_{1}} / 2 \\
0 & 1 & -\bar{\theta}_{1} \\
0 & 0 & 1
\end{array}\right) \operatorname{diag}\left(e^{-\theta_{2}}, 1, e^{\theta_{2}}\right)\left(\begin{array}{ccc}
1 & 0 & 0 \\
-\theta_{3} & 1 & 0 \\
-\theta_{4}+\theta_{3} \theta_{5} / 2 & -\theta_{5} & 1
\end{array}\right)
$$


Since the asymptotic conditions imply that $\chi$ and $\chi^{\prime}$ are the unit matrix at infinity, the matrix $\mathcal{G}$ must satisfy the condition $\Xi \mathcal{G}^{\dagger} \Xi=\mathcal{G}^{-1}$. This leads with (53) to

$$
e^{\theta_{2}}=\frac{1}{1-\theta_{1} \bar{\theta}_{1} / 2}, \quad \theta_{3}=i \overline{\theta_{1}}, \quad \theta_{4}=0, \quad \theta_{5}=\theta_{1} .
$$

The matrix $\mathcal{G}$ thus takes the form

$$
\mathcal{G}=\frac{1}{1-\theta_{1} \bar{\theta}_{1} / 2}\left(\begin{array}{ccc}
1 & i \theta_{1} & -i \theta_{1} \overline{\theta_{1}} / 2 \\
-i \overline{\theta_{1}} & 1+\theta_{1} \overline{\theta_{1}} / 2 & -\overline{\theta_{1}} \\
i \theta_{1} \bar{\theta}_{1} / 2 & -\theta_{1} & 1
\end{array}\right)
$$

If we transform an Ernst potential in the case $\Phi=0$, we end up with

$$
\Phi^{\prime}=-\frac{\theta_{1}}{\sqrt{2}} \frac{\theta_{1} \bar{\theta}_{1}\left(f^{2}+b^{2}\right) / 2-\left(1+\theta_{1} \overline{\theta_{1}} / 2\right) f+1-i b\left(1-\theta_{1} \overline{\theta_{1}} / 2\right)}{\left(1-\theta_{1} \bar{\theta}_{1} f / 2\right)^{2}+\left(\theta_{1} \bar{\theta}_{1} / 2\right)^{2} b^{2}} .
$$

We are interested in transformations which preserve the equatorial symmetry, i.e. $f(-\zeta)=f(\zeta), b(-\zeta)=$ $-b(\zeta)$ and $\Phi(-\zeta)=\bar{\Phi}(\zeta)$. This implies for (56) that $\theta_{1}$ must be real which rules out magnetic monopoles. We put $q=\theta_{1} / \sqrt{2}$ and sum up the results for the transformed potentials:

$$
\begin{aligned}
f^{\prime} & =\frac{\left(1-q^{2}\right)^{2} f}{\left(1-q^{2} f\right)^{2}+q^{4} b^{2}} \\
b^{\prime} & =\frac{\left(1-q^{4}\right) b}{\left(1-q^{2} f\right)^{2}+q^{4} b^{2}} \\
\Phi^{\prime} & =-q \frac{(1-f)\left(1-q^{2} f\right)+q^{2} b^{2}+i b\left(1-q^{2}\right)}{\left(1-q^{2} f\right)^{2}+q^{4} b^{2}} .
\end{aligned}
$$

The real parameter $q$ has to be in the region $0<|q|<1$, for $q>1$ the transformed spacetime would have a negative mass. The value $q=0$ corresponds to the untransformed solution. The above formulas imply that the functions $f^{\prime}, b^{\prime}$ and $\Phi^{\prime}$ are analytic where the original functions are analytic.

The metric function $k$ is invariant under the action of $S U(2,1)$ transformations. To determine the transformed metric function $a$, we consider the matrix $S$ in (18). If we go over from $2 \times 2$ matrices to $3 \times 3$ matrices according to the rule

$$
\left(\begin{array}{cc}
A_{11} & A_{12} \\
A_{21} & A_{22}
\end{array}\right) \rightarrow\left(\begin{array}{ccc}
A_{11} & 0 & A_{12} \\
0 & 1 & 0 \\
A_{21} & 0 & A_{22}
\end{array}\right)
$$

the matrix $\mathcal{G}$ acts on $S$ as on $\chi$. Thus we get with (55)

$$
\mathcal{S}_{12}^{\prime}=\frac{1}{\left(1-q^{2}\right)^{2}}\left(\mathcal{S}_{12}+2 i q^{2}-q^{4} \mathcal{S}_{21}\right)
$$

which is in accordance with (48). This implies with (111) for the function $a^{\prime}$

$$
a^{\prime}-a_{0}^{\prime}=-\frac{2}{\left(1-q^{2}\right)^{2}}\left(\mathcal{S}_{12}-q^{4} \mathcal{S}_{21}\right) .
$$

To determine $a_{0}^{\prime}$, one has to consider $\mathcal{S}_{12}$ and $\mathcal{S}_{21}$ on the axis. In the limit $\rho \rightarrow 0$, there is a nontrivial contribution from the quotient of theta functions in (20) which diverges as $1 / \rho$. Repeating the considerations of [27] in the calculation of $a_{0}$, one finds that the axis potentials can be expressed in terms of theta functions on the surface $\tilde{\Sigma}$ of genus $g-1$ given by $\tilde{\mu}^{2}=\prod_{i=1}^{g}\left(K-E_{i}\right)\left(K-\bar{E}_{i}\right)$. Denoting quantities on $\tilde{\Sigma}$ with a tilde, one has with [27] on the axis

$$
\frac{\mathcal{S}_{21}}{\mathcal{S}_{12}}=\frac{\tilde{\Theta}[\tilde{m}]\left(\tilde{u}+2 \tilde{\omega}\left(\infty^{-}\right)\right)}{\tilde{\Theta}[\tilde{m}]\left(\tilde{u}+2 \tilde{\omega}\left(\infty^{+}\right)\right)} .
$$


In the equatorially symmetric case, this quotient is identical to one since $2 \tilde{\omega}\left(\infty^{+}\right)$is a half period on $\tilde{\Sigma}$ (see [27]). Thus we have in this case

$$
a_{0}^{\prime}=a_{0} \frac{1+q^{4}}{\left(1-q^{2}\right)^{2}} .
$$

A well-known example is the Harrison transformation of the Schwarzschild solution which leads to the Reissner-Nordström solution. There we have with $r_{ \pm}=\sqrt{(\zeta \pm m)^{2}+\rho^{2}}$ the relations $f=\left(r_{+}+r_{-}-\right.$ $2 m) /\left(r_{+}+r_{-}+2 m\right), b=0$ which implies with (59)

$$
f^{\prime}=\frac{\left(r_{+}+r_{-}\right)^{2}-4 m^{2}}{\left(r_{+}+r_{-}+2 m^{\prime}\right)^{2}}, \quad \Phi^{\prime}=\frac{2 Q}{r_{+}+r_{-}+2 m^{\prime}},
$$

and $b^{\prime}=0$. We thus get a static solution with mass $m^{\prime}$ and charge $Q$ which are subject to the relation $m^{\prime 2}-Q^{2}=m^{2}$.

\subsection{Asymptotic behavior}

We assume that the asymptotic behavior of the original solution, which is read off on the axis, is of the form $f=1-2 M /|\zeta|, b=-2 J / \zeta^{2}$ and $\Phi=Q /|\zeta|-i J_{M} / \zeta^{2}$ plus terms of lower order in $1 /|\zeta|$ where $M$ is the Arnowitt-Deser-Misner mass, $J$ the angular momentum, $Q$ the electric charge and $J_{M}$ the magnetic moment. The same will hold for the Harrison transformed potentials. We find

$$
M^{\prime}=M \frac{1+q^{2}}{1-q^{2}}-\frac{2 q}{1-q^{2}} Q, \quad J^{\prime}=J \frac{1+q^{2}}{1-q^{2}}-\frac{2 q}{1-q^{2}} J_{M}
$$

and

$$
Q^{\prime}=Q \frac{1+q^{2}}{1-q^{2}}-\frac{2 q}{1-q^{2}} M, \quad J_{M}^{\prime}=J_{M} \frac{1+q^{2}}{1-q^{2}}-\frac{2 q}{1-q^{2}} J .
$$

It is interesting to note that the quantities $M^{2}-Q^{2}$ and $J^{2}-J_{M}^{2}$ are invariants of the transformation. They are related to the Casimir operator of the $S U(2,1)$-group. If the original solution was uncharged, the extreme relation $M^{\prime}= \pm Q^{\prime}$ is only possible in the limit $M=0$.

A further invariant is the combination $J_{M} M-J Q$ which is of importance in relation to the gyromagnetic ratio

$$
g_{M}=\frac{2 M J_{M}}{J Q} .
$$

Relation (66) implies that $g_{M}$ is equal to 2 if $Q=J_{M}=0$ and $q \neq 0$. Thus all solutions which can be generated via a Harrison transformation from solutions with vanishing electromagnetic fields as the Kerr-Newman family from Kerr have a gyromagnetic ratio of 2. Due to the invariance of $J_{M} M-J Q$ under Harrison transformations, a gyromagnetic ratio of 2 is not changed under the transformation.

\section{Charged disks}

In this section we will use the Harrison transformation (55) on the dust disk [15] to generate a disk solution to the Einstein-Maxwell equation. We discuss the metric, several interesting limiting cases and the energy-momentum tensor.

\subsection{Metric functions}

Since the metric function $e^{2 k}$ is invariant under Harrison transformations, we refer the reader to 18 for a discussion. The metric function $f^{\prime}$ in (57) is proportional to $f$, which implies that the transformed solution vanishes exactly where the original solution has zeros. Since the set of zeros of $f$ just defines the ergoregions, the transformed solution has the same ergoregions (if any) as the original solution. 
For small $q$, the functions $f$ and $b$ are essentially unchanged since they are quadratic in $q$. The electromagnetic potential $\Phi$ is in this limit with (59) of the form

$$
\Phi^{\prime}=-q(1-f+i b) .
$$

For larger $q,\left|f^{\prime}\right|$ becomes smaller near the origin. Since its asymptotic values are not changed, the growth rate towards infinity increases which is reflected by the mass formula [66). In the singular limit $q \rightarrow 1$, the function $f^{\prime}$ is zero for all finite values of $z$, but one at infinity. The behavior for $b^{\prime}$ is similar with the exception that $b^{\prime}$ is odd and zero at infinity. The function $a$ also becomes singular in the limit $q \rightarrow 1$ which is reflected by the diverging factor $1 /\left(1-q^{2}\right)^{2}$ and the constant $a_{0}$ (64) which just implies that one can no longer choose $a$ to be zero on the axis. In the metric function $g_{03}^{\prime}=-a^{\prime} f^{\prime}$, the factors $\left(1-q^{2}\right)^{2}$ just cancel and the function is only marginally changed with increasing $q$. The typical behavior of $g_{03}^{\prime}$ for values of $q$ with $0<|q|<1$ can be seen in Fig. 1. The metric function is an even function in $\zeta$ which

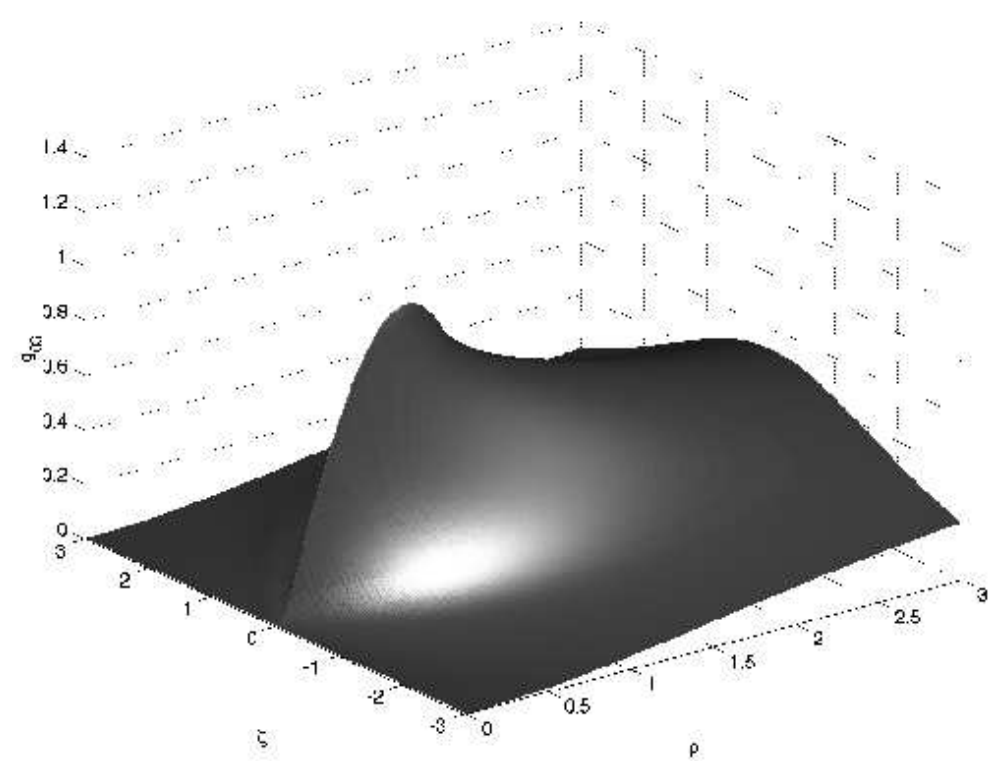

Figure 1: Metric function $g_{03}^{\prime}$ for $\epsilon=0.85, \gamma=0.95$ and $q=0.6$.

vanishes on the axis and at infinity. It is analytic except on the disk where the normal derivatives have a jump.

The electromagnetic potential tends to -1 in the limit $q \rightarrow 1$ for finite $\xi$, but is zero at infinity. The imaginary part is directly proportional to $b^{\prime}$ as can be seen from (58) and (559). We show a typical situation for values of $q$ with $0<|q|<1$ in Fig. 2 for the real part and in Fig. 3 for the imaginary part. The real part of $\Phi$ is an even function in $\zeta$ which vanishes at infinity and has discontinuous normal derivatives at the disk. The imaginary part of $\Phi$ is an odd function in $\zeta$ and has a jump at the disk. It vanishes at infinity.

Since $f^{\prime}$ has the same zeros as $f$, the transformed solution has a diverging central redshift if the untransformed has, i.e. the ultrarelativistic limits coincide. In the case $\gamma \neq 1$, one has a charged disk of finite extension with diverging central redshift. For $\gamma=1$, the solution in the exterior of the disk can be interpreted as an extreme Kerr-Newman metric which is obtained as a Harrison-transformed extreme Kerr metric.

In the Newtonian limit $\lambda \rightarrow 0$, one has $f=1+\lambda U_{N}$ and $b=\lambda^{\frac{3}{2}} \tilde{b}$ in lowest order. This implies with (57) to (59) for $1-q^{2}>>\lambda$

$$
f^{\prime}=1+\frac{1+q^{2}}{1-q^{2}} \lambda U_{N}, \quad b^{\prime}=\frac{1+q^{2}}{1-q^{2}} \lambda^{\frac{3}{2}} \tilde{b} \quad \Phi^{\prime}=-\frac{q}{1-q^{2}}\left(\lambda U_{N}+i \lambda^{\frac{3}{2}} \tilde{b}\right),
$$




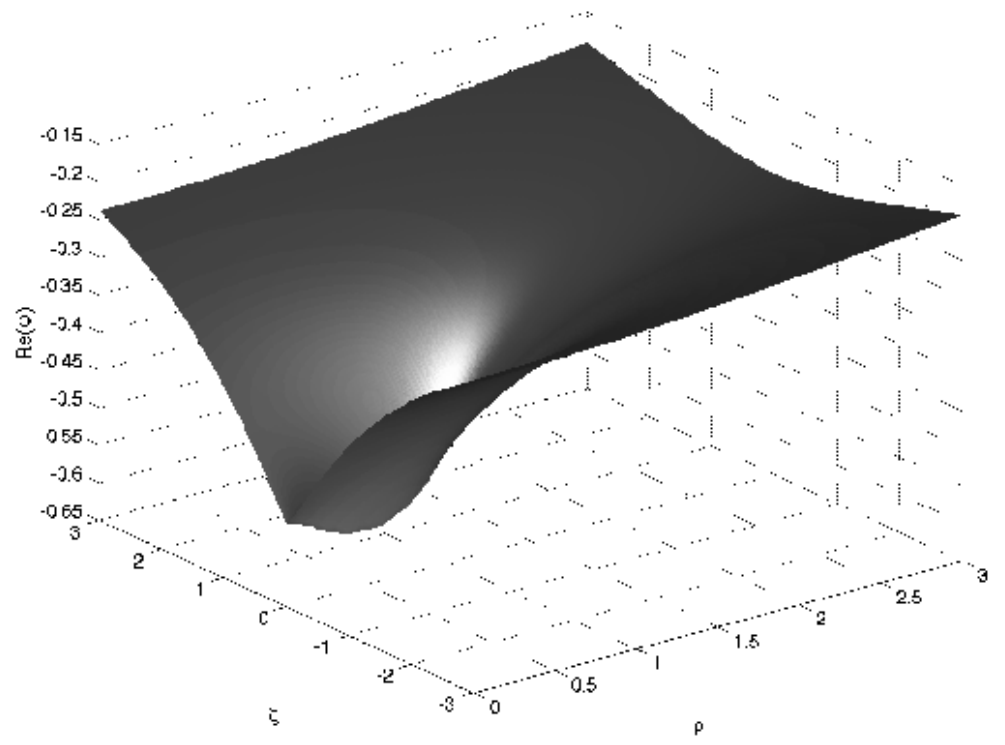

Figure 2: Real part of the electromagnetic potential $\Phi$ for $\epsilon=0.85, \gamma=0.95$ and $q=0.6$.

The transformed solution thus has the same Newtonian and post-Newtonian behavior as the original metric and in addition an electromagnetic field. The magnetic field is of order $\Omega^{3}$ as $b^{\prime}$.

Since the mass is of order $\lambda$ in the Newtonian limit, it is possible to have an extreme limit here with $M^{\prime}=Q^{\prime}$ as in the Reissner-Nordström solution. If we put $1-q^{2}=\kappa \lambda$ with $\kappa>0$, we get in the limit $\lambda \rightarrow 0$ for (57) and (59)

$$
f^{\prime}=\frac{\kappa^{2}}{\left(\kappa-U_{N}\right)^{2}} \quad \Phi^{\prime}=\frac{U_{N}}{\kappa-U_{N}},
$$

a static solution similar to the extreme Reissner-Nordström solution, but with a jump in the normal derivatives of the metric functions at the disk and non-vanishing $f^{\prime}$ at the origin. Since $U_{N}<0$ in the whole spacetime, the solution is regular in the exterior of the disk. Thus one gets a non-singular limit in the exterior of the disk for $q \rightarrow 1$ in this case.

In the static limit one has $b^{\prime}=\Im \Phi^{\prime}=0$, since both are proportional to $b$,

$$
f^{\prime}=\frac{\left(1-q^{2}\right) f}{\left(1-q^{2} f\right)^{2}}, \quad \Phi^{\prime}=-q \frac{1-f}{1-q^{2} f} .
$$

The Harrison-transformed static solution is thus again static with vanishing magnetic field but non-zero electric field.

\subsection{Disk and energy-momentum tensor}

It remains to be checked whether and in which range of the parameters the energy-momentum tensor (76) is physically acceptable. The above discussion of the metric indicates the extreme behavior of the metric functions for $q$ close to one. It is plausible that the matter in the disk which is in the present example the source of such an extreme metric will in general not be physically acceptable. There can be maximal $q$ smaller than 1 for given $\lambda$ and $\delta$ which limits the physical range of the parameters. The matter in the disk will be interpreted by following Ledvinka et al. $[5]$.

To discuss the energy-momentum tensor and the currents in the disk, it is helpful to use the algebraic relations (39) to (41) between the metric functions which exist at the disk, and which imply similar relations between the transformed potentials. With (30) and (31), we get for $S_{21}$

$$
\mathcal{S}_{21}=\mathcal{E} \overline{\mathcal{E}} S_{12}+i x_{0} f
$$




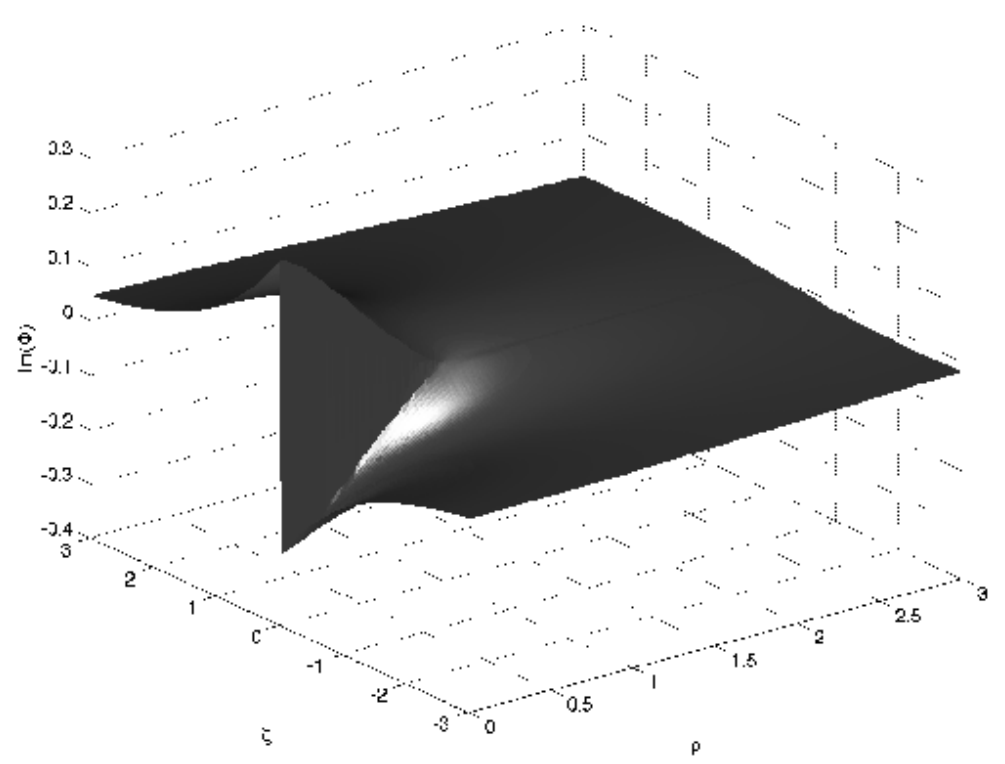

Figure 3: Imaginary part of the electromagnetic potential $\Phi$ for $\epsilon=0.85, \gamma=0.95$ and $q=0.6$.

and thus with (62) for the metric function $a^{\prime}$

$$
\left(1-q^{2}\right)^{2}\left(a-a_{0}\right)^{\prime}=\left(a-a_{0}\right)\left(1+q^{4} \frac{2}{\delta}\left(\frac{1}{\delta \lambda}\left(\frac{1 / \lambda^{2}+\delta}{\sqrt{1 / \lambda^{2}+\delta \rho^{2}}}-\frac{1}{\lambda}\right)+\alpha+\frac{\rho^{2}}{2}\right)\right),
$$

where $a_{0}^{\prime}$ is given by (64). With this function we can determine the angular velocity $\omega_{l}$ with respect to infinity of the locally non-rotating observers, for whom the metric is diagonal,

$$
\omega_{l}=-\frac{g_{03}}{g_{33}}=\frac{a e^{4 U}}{\rho^{2}-a^{2} e^{4 U}} .
$$

This quantity is a measure for the dragging of the inertial frames with respect to infinity due to the rotating matter in the disk. The dependence of $\omega_{l}$ on $\epsilon$ and $\gamma$ has been discussed in 18. As a function of $q$ it is monotonically decreasing as can be seen in Fig. [ The reason for this behavior is that the function $f^{\prime}$ tends to zero in the limit $q \rightarrow 1$ for finite $\rho, \zeta$ whereas $g_{03}^{\prime}$ changes shape but remains finite. Thus the overall behavior of $\omega_{l}$ is dominated by $f^{\prime}$. The deformation of the function $g_{03}^{\prime}$ via $q$ has however the consequence that $\omega_{l}$ has its maximum for large $q$ no longer at the center at the disk but near the rim. The energy-momentum tensor at the Harrison-transformed disk can be calculated via (42). Expressing the right-hand sides with the help of (57) and (58) via the original functions, we get

$$
\begin{aligned}
s_{1}^{\prime} & =\frac{\left(1-q^{2}\right)^{2}}{N^{2}}\left(\frac{\rho N}{2 Z} b_{\rho}-\left(1-q^{4} f^{2}+q^{4} b^{2}\right) f_{\zeta}+2 q^{4} f b b_{\zeta}\right), \\
s_{2}^{\prime} & =\frac{\rho}{2 f N}\left(\left(1-q^{4} f^{2}+q^{4} b^{2}\right) b_{\rho}+2 q^{4} b f f_{\rho}\right), \\
s_{3}^{\prime} & =-\frac{\rho^{3} N}{2\left(1-q^{2}\right)^{2} f^{2} Z} b_{\rho},
\end{aligned}
$$

where $N=\left(1-q^{2} f\right)^{2}+q^{4} b^{2}$.

Similarly a discontinuous electromagnetic tensor $F$ leads to a current density $J^{\alpha}$ via

$$
F_{; \beta}^{\alpha \beta}=\frac{1}{\sqrt{-\operatorname{det}\left(g_{\mu \nu}\right)}}\left(\sqrt{-\operatorname{det}\left(g_{\mu \nu}\right)} F^{\alpha \beta}\right)_{, \beta}=-4 \pi J^{\alpha} .
$$




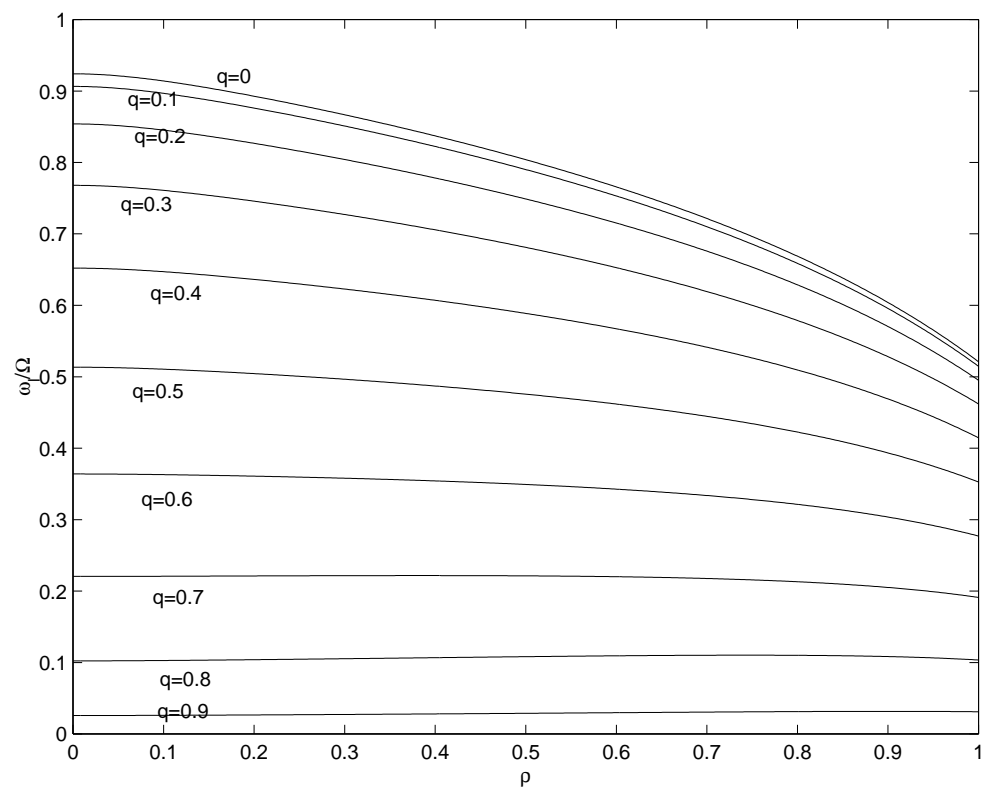

Figure 4: Angular velocity of the locally non-rotating observers with respect to infinity for $\epsilon=0.85$, $\gamma=0.95$, and $q=0, \ldots, 0.9$.

Contributions to $J$ arise only via the normal derivatives at the disk. We define the current density $j^{\alpha}$ in the disk as $s^{\alpha \beta}$ by the relation $J^{\alpha}=: e^{-2(k-U)} j^{\alpha} \delta(\zeta)$. The electromagnetic energy-momentum tensor does not produce a $\delta$-type contribution to the Einstein-Maxwell equations since $F$ is bounded at the disk. With (77) we get using equatorial symmetry (the derivatives are taken at $\zeta=0^{+}$)

$$
2 \pi j_{0}=-(\Re \Phi)_{\zeta}, \quad 2 \pi\left(j_{3}-a j_{0}\right)=-\frac{\rho}{f}(\Im \Phi)_{\rho} .
$$

This implies

$$
\begin{aligned}
2 \pi j_{0} & =\frac{q\left(1-q^{2}\right)}{N^{2}}\left(-\left(\left(1-q^{2} f\right)^{2}-q^{4} b^{2}\right) f_{\zeta}+2 q^{2} b\left(1-q^{2} f\right) b_{\zeta}\right) \\
2 \pi\left(j_{3}-a j_{0}\right) & =\frac{\rho q}{\left(1-q^{2}\right) N f}\left(2 q^{2} b\left(1-q^{2} f\right) f_{\rho}+\left(\left(1-q^{2} f\right)^{2}-q^{4} b^{2}\right) b_{\rho}\right) .
\end{aligned}
$$

The continuity equation (the Bianchi identity) at the disk $T_{; \nu}^{\mu \nu}=F^{\mu \nu} J_{\nu}=0$ leads to the condition

$$
g_{00, \rho} s^{00}+2 g_{03, \rho} s^{03}+g_{33, \rho} s^{33}=2\left(F_{10} j^{0}+F_{13} j^{3}\right) .
$$

We remark that one can substitute one of the equations (42) by (80) in the same way as one replaces one of the field equations by the covariant conservation of the energy momentum tensor in the case of three-dimensional perfect fluids. This makes it possible to eliminate $k_{\zeta}$ from (42) and to treat the energymomentum tensor at the disk purely on the level of the Ernst equation. It is straight forward to check the consistency of this approach with the help of (47). Thus one can solve boundary value problems of dust disks without using $k$.

We will interpret the matter in the disk as in [5] in two ways. One possible approach is to introduce observers for whom the tensor $s^{\alpha \beta}$ is diagonal ( $\phi$-isotropic observers or FIOs), i.e. we write $s^{\alpha \beta}$ in the form

$$
s^{\alpha \beta}=\sigma^{*} v^{\alpha} v^{\beta}+p^{*} w^{\alpha} w^{\beta},
$$


where $v$ and $w$ are the unit timelike respectively spacelike vectors $N_{v}\left(1,0, \omega_{\phi}\right)$ and $N_{w}(\kappa, 0,1)$ with $v_{\alpha} w^{\alpha}=0$. We get

$$
\omega_{\phi}=\frac{g_{33} s_{00}-g_{00} s_{33}+\sqrt{\left(g_{33} s_{00}-g_{00} s_{33}\right)^{2}+4\left(g_{03} s_{00}-g_{00} s_{03}\right)\left(g_{03} s_{33}-g_{33} s_{03}\right)}}{2\left(g_{03} s_{33}-g_{33} s_{03}\right)}
$$

and

$$
\kappa=-\frac{g_{03}+\omega_{\phi} g_{33}}{g_{00}+\omega_{\phi} g_{03}}
$$

We show $\omega_{\phi}$ for several values of $q$ in Fig. [5] It can be seen that $\omega_{\phi}$ decreases monotonically with $q$. For large $q$ the maximum of the angular velocity is near or at the rim of the disk in this example.

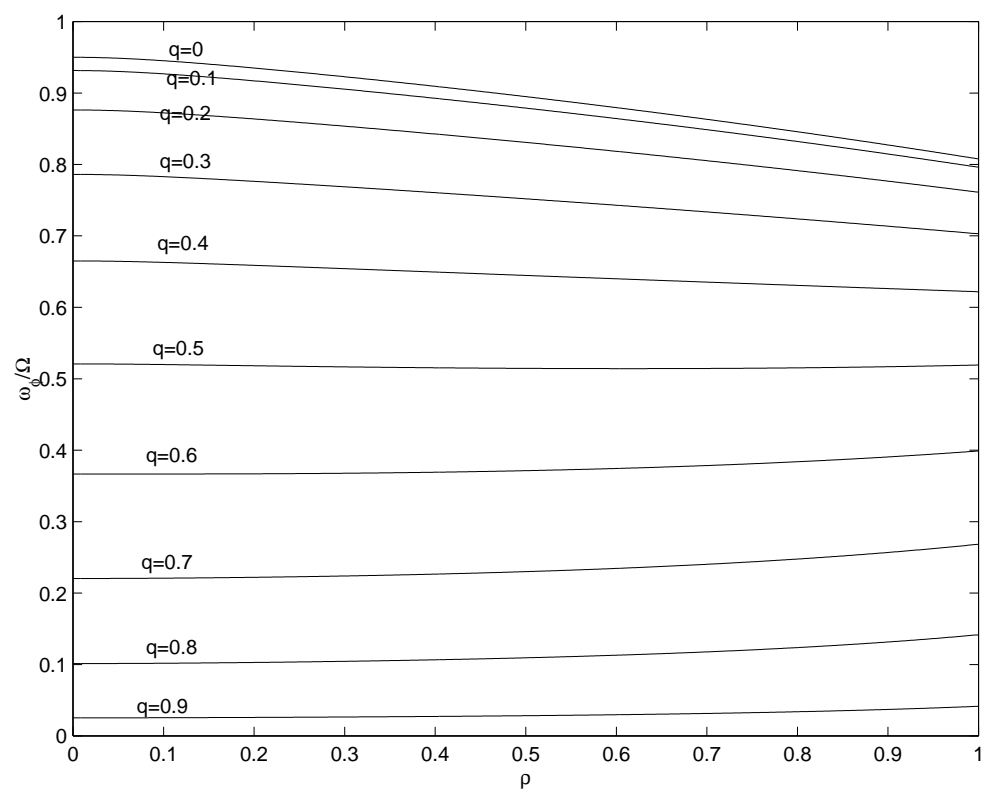

Figure 5: Angular velocity of the FIOs with respect to infinity for $\epsilon=0.85, \gamma=0.95$, and $q=0, \ldots, 0.9$.

If the energy conditions are satisfied which is the case here, the FIOs can interpret the matter in the disk as a fluid with a purely azimuthal pressure. Alternatively they can interpret it as being made up of two streams of counter-rotating pressureless matter, $S^{\alpha \beta}=\frac{1}{2} \sigma^{*}\left(U_{+}^{\alpha} U_{+}^{\beta}+U_{-}^{\alpha} U_{-}^{\beta}\right)$ with $\left(U_{ \pm}^{\alpha}\right)=N\left(1,0, \pm \Omega_{c}\right)$. The velocity $\Omega_{c} \rho$ is here below 1 (the speed of light). The matter streams will however not move on electro-geodesics unless $j_{\alpha} w^{\alpha}=0$, i.e. if there are no currents in the frame of the FIOs. However it can be seen in Fig 6 that there are in general currents in the frame of the FIOs. Thus an interpretation of the matter by the FIOs as freely moving charged particles is not possible.

A different approach is to interpret the matter in the disk as two streams of particles moving on electrogeodesics in the asymptotically non-rotating frame. To this end we make the ansatz

$$
s^{\alpha \beta}=\sigma_{m}^{+} U_{+}^{\alpha} U_{+}^{\beta}+\sigma_{m}^{-} U_{-}^{\alpha} U_{-}^{\beta}, \quad j^{\alpha}=\sigma_{e}^{+} U_{+}^{\alpha}+\sigma_{e}^{-} U_{-}^{\alpha}
$$

with $\left(U_{ \pm}^{\alpha}\right)=N_{ \pm}\left(1,0, \omega_{ \pm}\right)$. The angular velocity follows from the electro-geodesic equation for each component,

$$
\frac{1}{2} \sigma_{m}^{ \pm}\left(g_{00, \rho}+2 g_{03, \rho} \omega_{ \pm}+g_{33, \rho} \omega_{ \pm}^{2}\right)=\sigma_{e}^{ \pm}\left(A_{0, \rho}+A_{3, \rho} \omega_{ \pm}\right) .
$$

Such an interpretation is possible if the angular velocities are real. Moreover the velocities $\omega_{ \pm} \rho$ in the disk should be smaller than 1 to avoid superluminal velocities, and the energy densities have to be positive, 


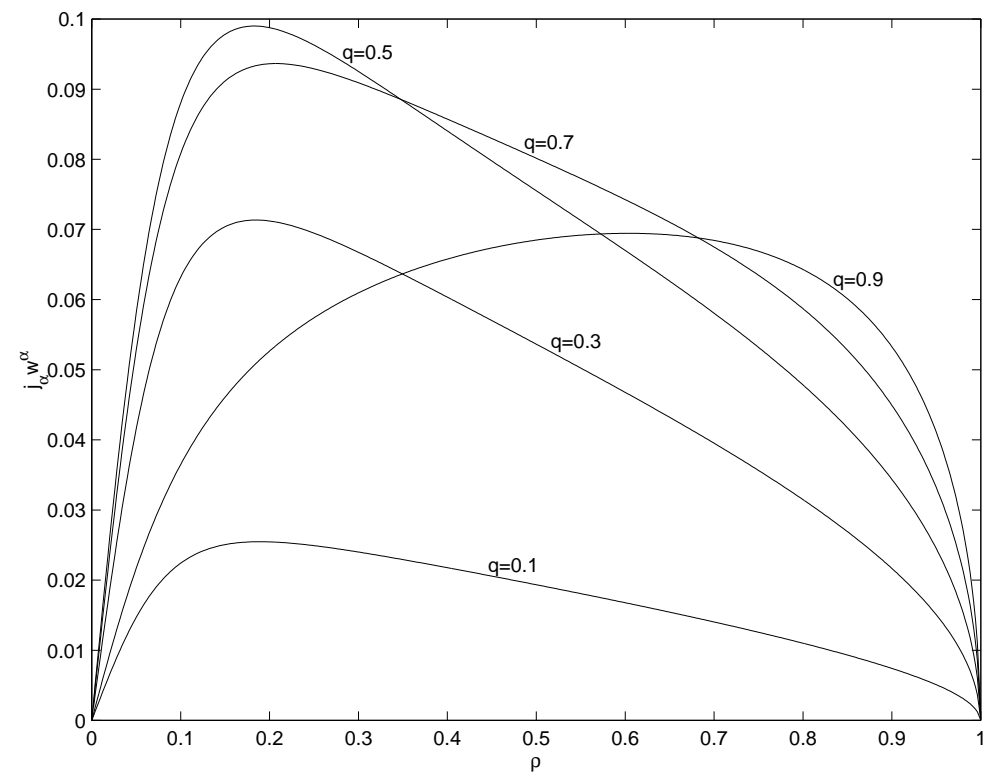

Figure 6: Currents in the frame of the FIOs for $\epsilon=0.85, \gamma=0.95$, and $q=0, \ldots, 0.9$.

which means we are not interested in tachyonic or otherwise exotic matter. Relation (84) leads to

$$
\begin{gathered}
\sigma_{e}^{+} N_{+}^{2}=\frac{j^{3}-\omega_{-} j^{0}}{\omega_{+}-\omega_{-}}, \quad \sigma_{e}^{-} N_{-}^{2}=\frac{j^{3}-\omega_{+} j^{0}}{\omega_{-}-\omega_{+}}, \\
\sigma_{m}^{+} N_{+}^{2}=\frac{s^{03}-\omega_{-} s^{00}}{\omega_{+}-\omega_{-}}, \quad \sigma_{m}^{-} N_{-}^{2}=\frac{s^{03}-\omega_{+} s^{00}}{\omega_{-}-\omega_{+}},
\end{gathered}
$$

and

$$
\omega_{-}=\frac{s^{33}-\omega_{+} s^{03}}{s^{03}-\omega_{+} s^{00}} .
$$

If we enter 85ith this, we obtain

$$
\omega_{ \pm}=\frac{-T_{2} \pm \sqrt{T_{2}^{2}-T_{1} T_{3}}}{T_{1}}
$$

with

$$
\begin{aligned}
T_{1} & =g_{33, \rho}-2 A_{3, \rho} \frac{j^{0} s^{03}-j^{3} s^{00}}{s^{03} s^{03}-s^{00} s^{33}} \\
T_{2} & =g_{03, \rho}-A_{0, \rho} \frac{j^{0} s^{03}-j^{3} s^{00}}{s^{03} s^{03}-s^{00} s^{33}}-A_{3, \rho} \frac{j^{3} s^{03}-j^{0} s^{33}}{s^{03} s^{03}-s^{00} s^{33}} \\
T_{3} & =g_{00, \rho}-2 A_{0, \rho} \frac{j^{3} s^{03}-j^{0} s^{33}}{s^{03} s^{03}-s^{00} s^{33}} .
\end{aligned}
$$

The densities then follow from (84) where the continuity equation (80) guarantees that this system can be solved.

Numerically one finds that the angular velocities are real, but in a wide range of the parameters there are negative energy densities and tachyonic behavior. Already in the uncharged case there are infinite velocities in strongly relativistic settings with negligible counter-rotation which are due to extrema of the metric function $g_{33}$ in the disk. In this case the quantity $T_{1}$ in (90) is zero which leads to a diverging 
$\omega_{-}$. Increasing $q$ only enhances this effect. The result is that an interpretation as non-tachyonic counterrotating matter on electro-geodesics with positive energy densities is only possible if $q, \epsilon$ and $\gamma$ are not too large. In other words large values of $q$ are in this setting only possible in post-Newtonian or nearly static situations. We show plots of the angular velocities $\omega_{ \pm}$in Fig. 7 for $\epsilon=0.36$ and $\gamma=.08$ where values of $q$ up to 0.75 are possible. The corresponding densities are given in Fig. 8 and the charge densities in

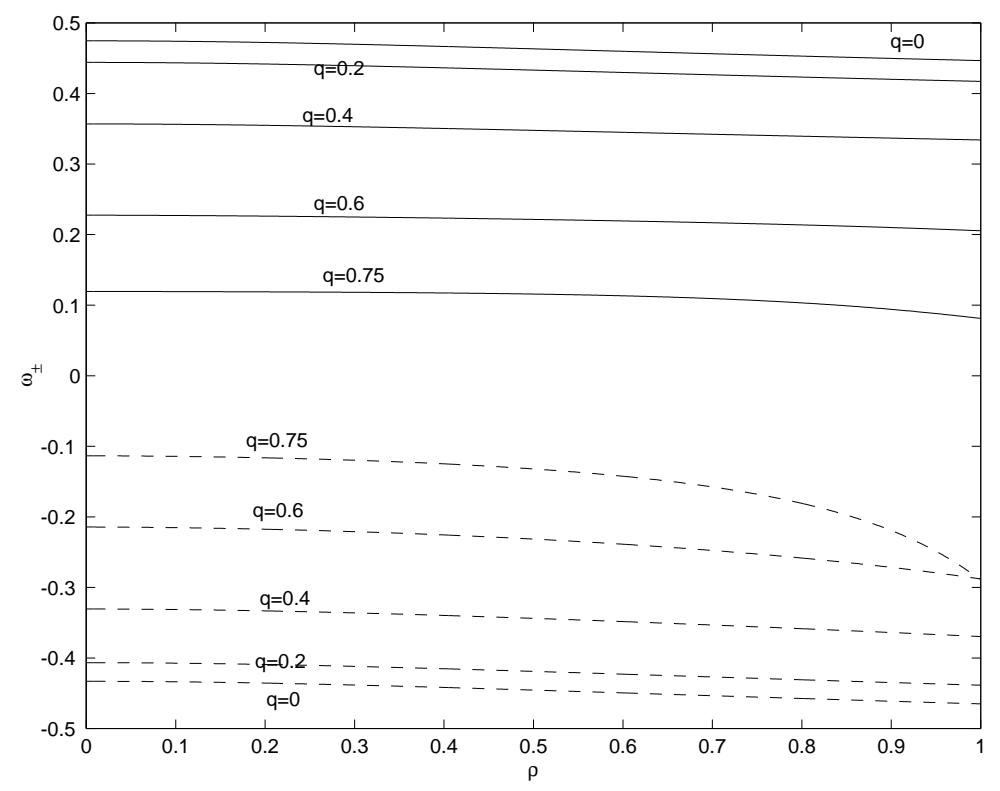

Figure 7: Angular velocities $\omega_{ \pm}$for $\epsilon=0.36, \gamma=0.08$, and $q=0,0.2, \ldots, 0.8$.

Fig. 9 The densities vanish always at the rim of the disk, the charge densities are identically zero for $q=0$. For larger values of $q$, the angular velocity $\omega_{-}$becomes bigger and bigger at the rim of the disk and finally diverges. The density $\sigma_{m}^{-}$is in this case negative in the vicinity of the rim of the disk.

\section{Conclusion}

In this article we discussed Harrison transformations of solutions to the stationary axisymmetric Einstein equations in vacuum to generate solutions to the corresponding Einstein-Maxwell equations. The case of hyperelliptic solutions to the Ernst equation was studied in detail. This led to a class of solutions to the Einstein-Maxwell system which contains the following degrees of freedom: a real-valued function $G$, a set of complex branch points $E_{i}$ and a parameter $q$ related to the total charge of the spacetime. Since the complete metric is given explicitly, one could in principle try to solve physically interesting boundary value problems as dust disks with a magnetic field within this class.

As a first step in this direction we have considered a Harrison transformation of a family of counterrotating dust disk solutions where the matter rotates in the frame of the FIOs on geodesics. The matter in the disk could be interpreted as moving on electro-geodesics in a certain range of the physical parameters, but in general not in the frame of the FIOs. As expected one cannot hope that a Harrison transformation preserves certain features of the matter as electro-geodesic motion in the FIO frame. If one wants to generate solutions with such properties or disks made up of charged pressureless particles without additional currents, one will have to solve the corresponding boundary value problem. Whether this will be possible within the class of Harrison transformed hyperelliptic solutions of the Ernst equation is an open question. For more general cases one will have to use Korotkin's theta functional solutions to the Einstein-Maxwell equations on three-sheeted surfaces, where however the powerful calculus of hyperelliptic surfaces can no longer be used. 


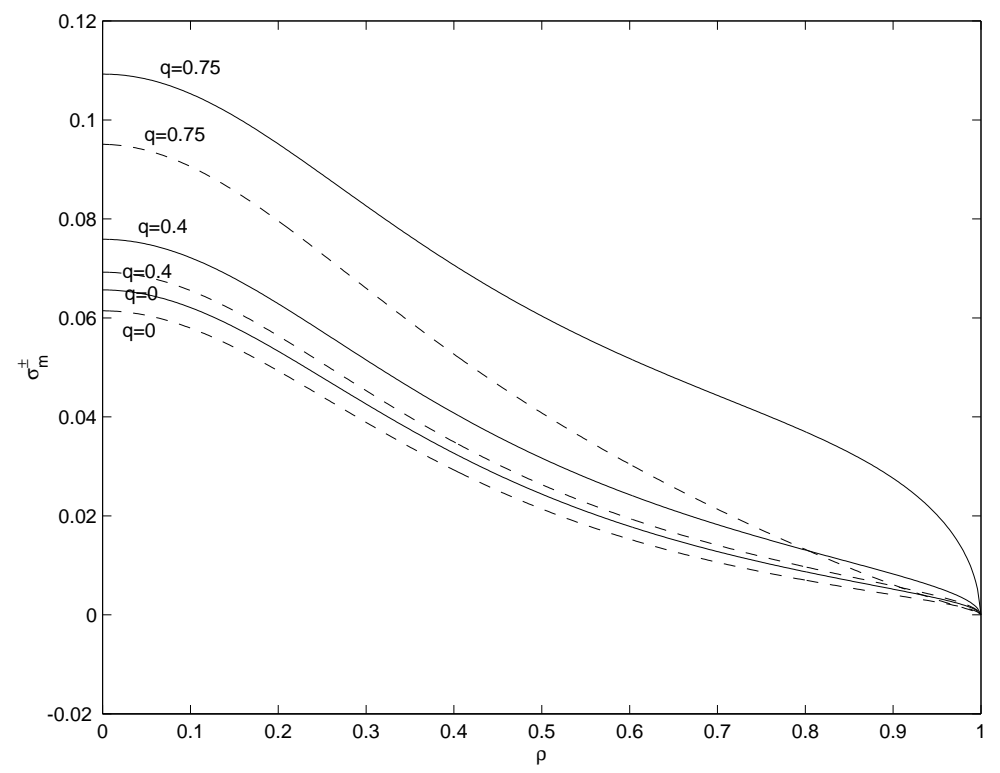

Figure 8: Matter densities $\sigma_{m}^{+}$and $\sigma_{m}^{-}$(dashed) for $\epsilon=0.36, \gamma=0.08$, and $q=0,0.4,0.8$.

Acknowledgement: I thank D. Maison, who interested me in the subject, for many helpful discussions and hints. This work was supported by the Schloessmann foundation.

\section{References}

[1] F. J. Ernst, Phys. Rev 168, 1415 (1968).

[2] D. Maison, Gen. Rel. Grav. 10, 717 (1979).

[3] J. Bičák, D. Lynden-Bell and J. Katz, Phys. Rev. D, 47, 4334 (1993) (1993).

[4] J. Bičák and T. Ledvinka, Phys. Rev. Lett., 71, 1669 (1993).

[5] T. Ledvinka, J. Bičák and M. Žofka, in Proceedings of 8th Marcel-Grossmann Meeting in General relativity, ed. by T. Piran (World Scientific: Singapore) (1999).

[6] P. Letelier, Phys. Rev. D, 60, 104042 (1999).

[7] J. Katz, J. Bičák and D. Lynden-Bell, Class. Quant. Grav., 16 , 4023 (1999).

[8] G. Neugebauer and D. Kramer, Ann. Phys. (Leipzig), 24, 62 (1969).

[9] W. Kinnersley, J. Math. Phys., 14, 651 (1973).

[10] Y. Tanabe, Prog. Theor. Phys., 57, 840 (1977).

[11] D. Maison, Nonlinear equations in classical and quantum field theory (Meudon/Paris, 1983/1984), Lecture Notes in Phys. 226, (Springer: Berlin) 125-139 (1985).

[12] P. Breitenlohner, G. Gibbons and D. Maison, Comm. Math. Phys., 120, 295 (1988).

[13] B. Harrison, J. Math. Phys. 9, 1744 (1968).

[14] D. A. Korotkin, Theor. Math. Phys. 77, 1018 (1989). 


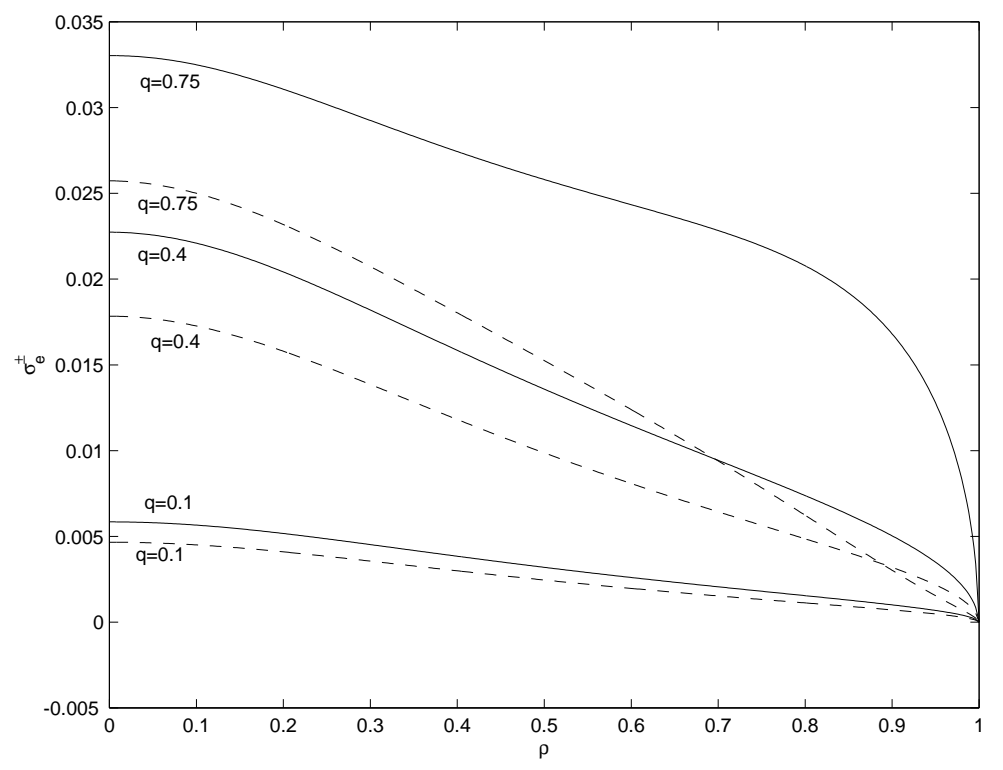

Figure 9: Charge densities $\sigma_{e}^{+}$and $\sigma_{e}^{-}$(dashed) for $\epsilon=0.36, \gamma=0.08$, and $q=0,0.4,0.8$.

[15] C. Klein and O. Richter, Phys. Rev. Lett., 83, 2884 (1999).

[16] J. M. Bardeen and R. V. Wagoner, Ap. J., 167, 359 (1971).

[17] G. Neugebauer and R. Meinel, Phys. Rev. Lett., 75, 3046 (1995).

[18] J. Frauendiener and C. Klein, Phys. Rev. D 63, 064033 (2001).

[19] C. Klein, Phys. Rev. D 63, 084025 (2001).

[20] D. Kramer, H. Stephani, E. Herlt and M. MacCallum, Exact Solutions of Einstein's Field Equations, Cambridge: CUP (1980).

[21] F. J. Ernst, Phys. Rev, 167, 1175 (1968).

[22] D. Maison, Phys. Rev. Lett., 41, 521 (1978).

[23] V. A. Belinski and V. E. Zakharov, Sov. Phys.-JETP, 48, 985 (1978).

[24] P. Breitenlohner and D. Maison, Ann. Inst. H. Poincare, 46, 215 (1987).

[25] D. Korotkin, Class. Quant. Grav., 10, 2587 (1993).

[26] J. D. Fay, Theta-functions on Riemann surfaces, Lect. Notes in Math. 352, Springer (1973).

[27] C. Klein and O. Richter, Phys. Rev. D, 58, CID 124018 (1998).

[28] E.D. Belokolos, A.I. Bobenko, V.Z. Enolskii, A.R. Its and V.B. Matveev, Algebro-Geometric Approach to Nonlinear Integrable Equations, Berlin: Springer, (1994).

[29] T. Morgan and L. Morgan, Phys. Rev., 183, 1097 (1969); Errata: 188, 2544 (1969).

[30] W. Israel, Nuovo Cimento 44B 1 (1966); Errata: Nuovo Cimento, 48B, 463 (1967). 